\title{
Cyclodextrin:A promising candidate in enhancing oral bioavailability of poorly water soluble drugs
}

\begin{abstract}
Most of the drugs administered through oral route have poor aqueous solubility and dissolution rate. Cyclodextrin and its derivates represent as pharmaceutical adjuvants to overcome this challenges and helps in development of stable formulation with enhanced bioavailability. Cyclodextrins are unique structure with versatile physicochemical properties which aids the pharmaceutical scientists to overcome drug delivery challenges for poorly aqueous soluble drugs. Cyclodextrin and its derivates are widely useful as solubilizers, assisting in preparation of various dosage forms such as liquid oral, solid, and parenteral preparations. Cyclodextrins interacts with appropriately sized guest molecules to form inclusion complex and enhance the aqueous solubility, physical chemical stability, and bioavailability of drugs. Through the various reported literatures, the review highlights the concept of cyclodextrin and its derivatives in enhancing solubility and bioavailability of poorly aqueous soluble drugs.
\end{abstract}

Keywords: cyclodextrin, oral delivery, bioavailability, solubility
Volume 3 Issue 3 - 2017

\section{Pankaj M Maheriya}

Department of Pharmaceutical Research and Development,

India

Correspondence: Pankaj M Maheriya, Department of Pharmaceutical Research and Development, Piramal Enterprises Limited, Hiranandani Light hall, Shaki Vihar Road, Mumbai-400072, Maharashtra, India, Tel 9|+8108765890, Email pankaj.maheriya@gmail.com

Received: September 21, 2016 | Published: April 26, 2017
Abbreviations: BCS, biopharmaceutical classification system; CDs, cyclodextrins

\section{Introduction}

Oral bioavailability of poorly aqueous soluble drugs remains one of the most challenging aspects for researchers in formulation development of dosage forms. Nearby, $70 \%$ of existing new drug molecules are poorly aqueous soluble and require a suitable candidate for enhancing oral bioavailability and solubility. Drugs having the water solubility of $<10 \mathrm{mg} / \mathrm{ml}$ over the $\mathrm{pH}$ range of $1-7$ at $37^{\circ} \mathrm{C}$ show bioavailability issues. ${ }^{1}$ According to Biopharmaceutical Classification System (BCS), drugs which are poorly soluble but highly permeable falls under BCS class II category. These poorly aqueous soluble drug molecules exhibit slow drug absorption leading to poor and erratic bioavailability and finally causes GI mucosal toxicity. Low solubility, poor dissolution rate and compromised oral absorption are the major problems of BCS Class II drugs and hence enhancing solubility of such actives is a major challenge for the pharmaceutical and academic researchers. ${ }^{2,3}$ Cyclodextrins (CDs) represents one of the pharmaceutical excipient in overcoming this challenge. CDs are molecules of natural origin, discovered earlier by Villiers in 1891 . The interest in application of CDs was later on studied by Austrian microbiologist Franz Schardinger in the twentieth century which became the most important topic of interest in pharmaceutical and other fields since from late 1970 s to later on. ${ }^{2}$ He described about two crystalline compounds isolated from bacterial digest of potato starch called $\alpha$-dextrin and $\beta$-dextrin which were later and now called as $\alpha-\mathrm{CD}$ and $\beta-\mathrm{CD} .{ }^{4}$ Over the span of time, CDs have created a quality platform for various applications like increasing drug solubility and stability, ${ }^{4}$ masking odors and tastes, ${ }^{5}$ enhancing drug absorption, ${ }^{6}$ controlling drug release profiles, ${ }^{7}$ alleviating local and systemic toxicity, ${ }^{8}$ and improving drug permeability across biological barriers. ${ }^{9}$ CDs containing formulations have been delivered through various delivery systems like oral, ocular, nasal, dermal and rectal. ${ }^{10-13}$ From application point of view, CDs offers various advantages like non-toxic, low cost, safety (recognized by safety health authorities) and easily available. ${ }^{14}$ Various published reports demonstrate wide application of CDs to enhance oral bioavailability of poorly aqueous soluble drugs. ${ }^{15,16}$

\section{Structure of CDs}

CDs are cyclic oligosaccharides obtained from starch degradation by cycloglycosyl transferase amylases produced by various bacilli (Bacillus macerans and B circulans). Depending on the exact reaction conditions, three main types of CDs are obtained, $\alpha-, \beta-$, and $\gamma-C D$, each comprises six to eight dextrose units respectively. ${ }^{17} \mathrm{CDs}$ are ring molecules which lack free rotation at the level of bonds between glucopyranose units, they are not cylindrical rather they are toroidal or cone shaped. CDs consists of hollow tapered cavity consist of $0.79 \mathrm{~nm}$ in depth in which the active molecule is incorporated. The primary hydroxyl groups are located on the narrow side whereas the secondary groups are on the wider side. ${ }^{14}$ The properties of CDs can be modified by substituting different functional groups on the CDs rim. Substituting the hydroxyl group of $\mathrm{CD}$ by chemical and enzymatic reactions by variety of substituting groups like hydroxypropyl-, methyl-, carboxyalkyl-, thio-, tosyl-, amino-, maltosyl-, glucosyl-, and sulfobutyl -ether-groups to $\beta-\mathrm{CD}$ can increase the solubility. Solubility of nonpolar solutes occurs due to the nonpolar nature (lipophilic) of the internal cavity of CDs whereas, the polar nature (hydrophilic) of CDs external part helps in solubilising the CDs and drug in aqueous solution. Due to this characteristics nature, CDs have attained a great interest as a solubilising candidate and has overcome the biopharmaceutical deficiencies of various drugs in the recent years. ${ }^{14,17-19} \mathrm{CDs}$ are widely soluble in some polar, aprotic solvents, but insoluble in most organic solvents. ${ }^{20}$ Although, CDs exhibit higher solubility in some of the organic solvents than in water, inclusion complexes do not take place in non-aqueous solvents because of the increased affinity of guest molecule for the solvent compared to its affinity for water. ${ }^{20}$ Strong acids such as hydrochloric acid and sulfuric acid can hydrolyze CDs. This hydrolysis rate depends upon 
temperature and concentration of the acid. CDs are stable against bases. ${ }^{20}$ The hydrophobic cavity in CDs can partially accommodate low molecular lipophilic drug molecule and polymers. ${ }^{21}$ Hydrophilic drug-CD complexes are formed by inclusion of lipophilic drug or lipophilic drug molecule in the central cavity. The lipophilic cavity thus protects the lipophilic guest molecule from aqueous environment, while the outer polar surface of the $\mathrm{CD}$ provides the solubilizing effect. $^{22}$

\section{$\beta-C D$ derivative in oral delivery}

Certain CDs limit its application in pharmaceuticals due to its low solubility and poor safety. One of them includes $\beta-C D$ which shows low solubility and produces haemolytic activity and strong irritancy. However, some $\beta-C D$ derivatives can overcome these shortcomings. Nevertheless, owing to its low price $\beta-C D$ derivatives are widely used in pharmaceutically marketed formulations. ${ }^{23}$ The solubility of $\beta-\mathrm{CD}$ in water is relatively low (approximately $18.554 \mathrm{mg} / \mathrm{mL}$ at $25^{\circ} \mathrm{C}$ ), whereas its derivative hydroxypropyl $-\beta-$ Cyclodextrin has a higher aqueous solubility (approx. $60055 \mathrm{mg} / \mathrm{mL}$ at $25^{\circ} \mathrm{C}$ ). Based on published reports, Hydroxypropyl- $\beta-$ cyclodextrin is widely used derivative of $\beta-C D$ in improving the solubility of hydrophobic drugs with its better aqueous solubility and higher safety. ${ }^{24}$ Table 1 represents the natural CDs and its available derivatives. Various reports have been published on $\beta-\mathrm{CD}$ as a host in inclusion complexes with the guest molecules and have been patent. ${ }^{25,26}$ Inclusion complex involves stoichiometric molecular phenomenon. CDs as a host forms hydrophilic inclusion complexes (When this cavity is filled with the molecule of another substance, it is called an inclusion complex) with the hydrophobic drug moieties (guest) by incorporating the guest molecule into the internal cavity in the ratio of 1:1 and thereby altering the physicochemical properties of active molecule without any change in the intrinsic properties of active molecule. ${ }^{3,27} \mathrm{Such}$, changes in the physicochemical properties of the drug molecules such as solubility, dissolution rate, stability and bioavailability ${ }^{28}$ contributes CDs as an apt candidate for oral drug delivery in the pharmaceutical field. Figure 1 depicts the guest-host inclusion complexes formation. Drug/CD inclusion complexes are usually prepared by simple unit processes such as precipitation, kneading, solvent evaporation, lyophilization and spray drying of solutions or suspensions of the components. ${ }^{29,30}$ The binding forces of the guest molecule within these inclusion complexes include hydrophobic, vander Waals, hydrogen bonding or dipole interaction. ${ }^{31,32}$

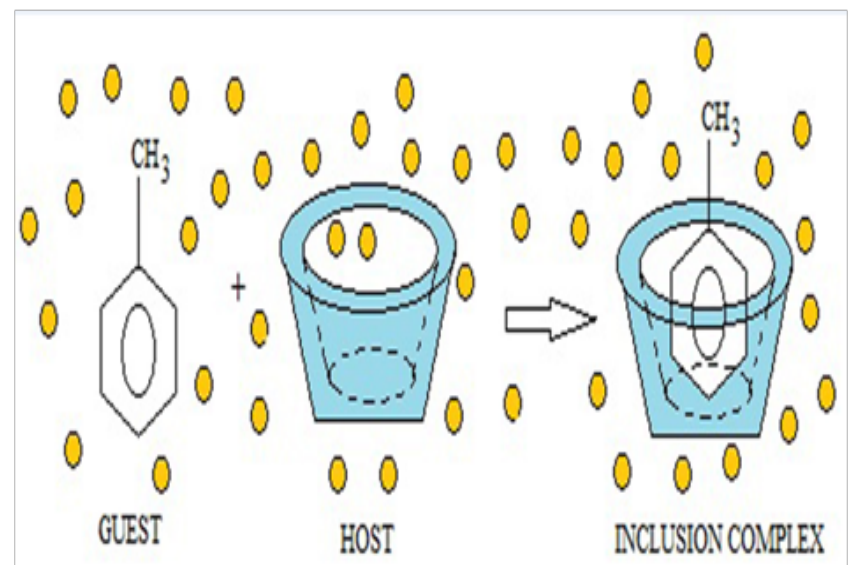

Figure I Depicts the guest-host inclusion complexes formation.
Focusing on hydroxypropyl- $\beta-$ cyclodextrin as a derivative of $\beta$ $\mathrm{CD}$ in enhancing solubility and bioavailability, various researchers have focus on its application in development of solid oral dosage forms. Cappello et al. ${ }^{21}$ prepared tablet for the buccal delivery of the poorly soluble drug carvedilol containing poly (ethyleneoxide) as bioadhesive material for sustained-release and hydroxypropyl- $\beta$-cyclodextrin as modulator for enhancing drug release. The results confirmed that the prepared hydroxypropyl- $\beta$-cyclodextrin containing tablets showed increase in drug dissolution. ${ }^{33}$ Nalluri et al. ${ }^{34}$ prepared nimesulide and meloxicam-hydroxypropyl- $\beta$-cyclodextrin binary systems to improve the pharmaceutical properties of both the drugs via inclusion complexation. The prepared formulations showed increase in aqueous solubility and dissolution properties of nimesulide and meloxicam by inclusion complexation with hydroxypropyl- $\beta-$ cyclodextrin. ${ }^{34}$ Vyas et al. ${ }^{35}$ developed a novel technique using hydroxypropyl- $\beta$-cyclodextrin and chitosan nanocarriers for effective delivery of poorly water soluble drug simvastatin. The results revealed that increase in the amount of hydroxypropyl- $\beta$ - cyclodextrin increased the solubilization of simvastatin. ${ }^{35}$ Wang and his colleagues formulated modified-release solid oral dosage form (bilayer tablet) of poorly water soluble drug nifedipine by hybridization of hydroxypropyl- $\beta$-cyclodextrin and Hydroxypropylcellulose. The results showed that hydroxypropyl- $\beta-$ cyclodextrin with Hydroxypropylcellulose can serve as immediate release carrier along with enhancing the oral bioavailability. ${ }^{36}$ Hamza et al..$^{37}$ develop double-layer tablets of lornoxicam by direct compression method. An amorphous, freeze-dried inclusion complex of lornoxicam with hydroxypropyl- $\beta$-cyclodextrin was present in 1:2 (drug/cyclodextrin) molar ratio in development of fast-release layer to enhance the dissolution of lornoxicam in the stomach and to assure rapid onset of its analgesic effect whereas, the sustained release layer was formed by using Xanthan gum. The results revealed that complex of hydroxypropyl- $\beta$-cyclodextrin with lornoxicam enhanced the dissolution rate of lornoxicam in acidic medium. ${ }^{37}$ Gidwani \& Vyas $^{38}$ prepared sustained release matrix tablets of a poorly soluble anticancer drug altretamine by fusion method using cyclodextrin complexation approach. The prepared binary system of Altretaminehydroxypropyl- $\beta$-cyclodextrin was developed to improve the aqueous solubility of drug. The application of hydroxypropyl- $\beta-$ cyclodextrin showed improved solubility of Altretamine by complexation approach. ${ }^{38}$ Huang et al. ${ }^{19}$ prepared inclusion complex of apigeninhydroxypropyl- $\beta$-cyclodextrin by using supercritical antisolvent process using N,N-dimethyl formamide as solvent and carbon dioxide as antisolvent for the enhancement of dissolution and bioavailability. The mole ratio of apigenin and hydroxypropyl- $\beta$-cyclodextrin was kept 1:1. In vitro study showed that the dissolution rate of the apigeninhydroxypropyl- $\beta-$ cyclodextrin inclusion complex was about 7.60 times higher than that of the raw apigenin whereas, in vivo study showed that the bioavailability of the inclusion complex increased by 6.45 times compared with that of the raw apigenin. Hence, the results showed that the prepared apigenin-hydroxypropyl- $\beta-$-cyclodextrin inclusion complex serves as a potential approach for new oral therapeutic agent formulation. ${ }^{19}$ Miyake et al. ${ }^{39}$ improved solubility, dissolution rate, and oral bioavailability of rutin by complexation with 2 -hydroxypropyl- $\beta$-cyclodextrin. The in vivo absorption study revealed that hydroxypropyl- $\beta-$ cyclodextrin increased the oral bioavailability of rutin from the gastrointestinal tracts due to increase in solubility, faster dissolution rate, and gastrointestinal stability. Hydroxypropyl- $\beta$-cyclodextrin showed a significant advantage with respect to providing high aqueous solubility while maintaining a lack of toxicity in oral pharmaceutical preparations of rutin. ${ }^{39}$ Choudhury et al..$^{40}$ improved solubility and bioavailability of poorly water 
soluble antiepileptic drug carbamazepine by complexation with $2-$ hydroxypropyl- $\beta-$ cyclodextrin (1:1 complex). The results concluded that oral bioavailability of carbamazepine was improved by using 2-hydroxypropyl- $\beta$-cyclodextrin through inclusion complex approach. $^{40}$

Table I Cyclodextrins and its derivatives Stella VJ et al..$^{33}$

\begin{tabular}{|c|c|c|}
\hline Cyclodextrins & $\mathbf{R}$ & $\mathbf{N}$ \\
\hline$\alpha-$ Cyclodextrin & $\mathrm{H}$ & 4 \\
\hline$\beta-$ Cyclodextrin & $\mathrm{H}$ & 5 \\
\hline$\gamma$-Cyclodextrin & $\mathrm{H}$ & 6 \\
\hline Carboxymethyl- $\beta-$ Cyclodextrin & $\mathrm{CH}_{2} \mathrm{CO}_{2} \mathrm{H}$ or $\mathrm{H}$ & 5 \\
\hline $\begin{array}{l}\text { Carboxymethyl-Ethyl- } \beta \text { - } \\
\text { Cyclodextrin }\end{array}$ & $\mathrm{CH}_{2} \mathrm{CO}_{2} \mathrm{H}, \mathrm{CH}_{2} \mathrm{CH}_{3}$ or $\mathrm{H}$ & 5 \\
\hline Diethyl- $\beta-$ Cyclodextrin & $\mathrm{CH}_{2} \mathrm{CH}_{3}$ or $\mathrm{H}$ & 5 \\
\hline Dimethyl- $\beta-$ Cyclodextrin & $\mathrm{CH}_{3}$ or $\mathrm{H}$ & 5 \\
\hline Glucosyl- $\beta-$ Cyclodextrin & Glucosyl or H & 5 \\
\hline Hydroxybutenyl- $\beta-$ Cyclodextrin & $\mathrm{CH}_{2} \mathrm{CH}\left(\mathrm{CHCH}_{2}\right) \mathrm{OH}$ or $\mathrm{H}$ & 5 \\
\hline Hydroxyethyl- $\beta-$ Cyclodextrin & $\mathrm{CH}_{2} \mathrm{CH}_{2} \mathrm{OH}$ or $\mathrm{H}$ & 5 \\
\hline Hydroxypropyl- $\beta-C y c l o d e x t r i n$ & $\mathrm{CH}_{2} \mathrm{CHOHCH}_{3}$ or $\mathrm{H}$ & 5 \\
\hline Hydroxypropyl- $\gamma-$ Cyclodextrin & $\mathrm{CH}_{2} \mathrm{CHOHCH}_{3}$ or $\mathrm{H}$ & 6 \\
\hline Maltosyl- $\beta-$ Cyclodextrin & Maltosyl or $\mathrm{H}$ & 5 \\
\hline Methyl- $\beta-$ Cyclodextrin & $\mathrm{CH}_{3}$ or $\mathrm{H}$ & 5 \\
\hline Random Methyl- $\beta-$ Cyclodextrin & $\mathrm{CH}_{3}$ or $\mathrm{H}$ & 5 \\
\hline Sulfobutylether- $\beta-$ Cyclodextrin & $\left(\mathrm{CH}_{2}\right)_{4} \mathrm{SO}_{3} \mathrm{Na}$ or $\mathrm{H}$ & 5 \\
\hline
\end{tabular}

\section{Conclusion}

CDs and CDs derivates have attained a promising platform in formulation development of poorly aqueous soluble drugs by enhancing solubility and bioavailability. Various CDs derivates have been pharmaceutical approved for their application in formulation development of different dosage forms and has been emerging with new applications for various delivery system. CDs and CDs derivates will serve as promising candidate in overcoming the challenges of poor solubility of novel and existing drugs and help the researchers and academicians in building up the platform in the formulation of novel delivery systems with improved solubility of drugs.

\section{Acknowledgements}

None.

\section{Conflict of interest}

The author declares no conflict of interest.

\section{References}

1. Semalty A. Cyclodextrin and phospholipid complexation in solubility and dissolution enhancement: a critical and meta-analysis. Expert Opin Drug Deliv. 2014;11(8):1255-1272.

2. Vakani S, Kajwe A, Suvarna V, et al. Influence of auxiliary agents on solubility and dissolution profile of repaglinide with hydroxypropyl- $\beta$ cyclodextrin:inclusion complex formation and its solid-state characterization. J Incl Phenom Macrocycl Chem. 2015;83(3):239-250.
3. Loftsson T, Moya Ortegaa MD, Alvarez Lorenzob C, et al. Pharmacokinetics of cyclodextrins and drugs after oral and parenteral administration of drug/cyclodextrin complexes. J Pharm and Pharmacol. 2015;68(5):544-555.

4. Brewster ME, Loftsson T. Cyclodextrins as pharmaceutical solubilizers. ¡Adv Drug Deliv Rev. 2007;59(7):645-666.

5. Patel A, Vavia PR. Preparation and evaluation of Taste Masked Famotidine Formulation Using Drug/ $\beta$-cyclodextrin/Polymer Ternary Complexation Approach. AAPS Pharm SciTech. 2008;9(2):544-550.

6. Carrier RL, Miller LA, Ahmed I. The utility of cyclodextrins for enhancing oral bioavailability. J Control Release. 2007;123(2):78-99.

7. Hirayama F, Uekama K. Cyclodextrin-based controlled drug release system. Adv Drug Deliv Rev. 1999;36(1):125-141.

8. Davis ME, Brewster ME. Cyclodextrin-based pharmaceutics: past, present, and future. Nat Rev Drug Discov. 2004;3(12):1023-1035.

9. Loftsson T, Brewster ME. Pharmaceutical applications of cyclodextrins: effects on drug permeation through biological membranes. J Pharm Pharmacol. 2011;63(9):1119-1135.

10. Loftsson T, Stefánsson E. Cyclodextrins in Eye Drop Formulations. Journal of Inclusion Phenomena. 2002;44(1):23-27.

11. Matsuda H, Arima H. Cyclodextrins in transdermal and rectal delivery. Adv Drug Deliv Rev. 1999;36(1):80-99.

12. Loftsson T, Järvinen T. Cyclodextrins in ophthalmic drug delivery. $A d v$ Drug Deliv Rev. 1999;36(1):59-79.

13. Merkus FW, Verhoef JC, Marttin E, et al. Cyclodextrins in nasal drug delivery. Adv Drug Deliv Rev. 1999;36(1):41-57.

14. D Duchene, G Ponchel, D Wouessidjewe. New developments in improvement of drug solubility and availability by cyclodextrins. Proceedings of the ninth international symposium on cyclodextrins. 1999;239-246.

15. Dhanaraju M, Kumaran KS, Baskaran T, et al. Enhancement of bioavailability of griseofulvin by its complexation with $\beta$-cyclodextrin. Drug Dev Ind Pharm. 1998;24(6):583-587.

16. Uekama, K, Otagiri M, Uemura Y, et al. Improvement in oral bioavailability of prednisolone by $\beta$-cyclodextrin complexation in humans. $J$ Pharmacobiodyn. 1983;6(2):124-127.

17. Olesen NE, Westh P, Holm R. Displacement of Drugs from Cyclodextrin Complexes by Bile Salts: A Suggestion of an Intestinal Drug-Solubilizing Capacity from an In vitro Model. J Pharm Sci. 2016;105(9):2640 2647.

18. Dora CP, Trotta F, Kushwah V, et al. Potential of erlotinib cyclodextrin nanosponge complex to enhance solubility, dissolution rate, in vitro cytotoxicity and oral bioavailability. Carbohydr Polym. 2016;137:339349

19. Huang Y, Zu Y, Zhao X, et al. Preparation of inclusion complex of apigenin-hydroxypropyl- $\beta$-cyclodextrin by using supercritical antisolvent process for dissolution and bioavailability enhancement. Int J Pharm. 2016;511(2):921-930.

20. Shimpi S, Chauhan B, Shimpi P. Cyclodextrins: Application in different routes of drug administration. Acta Pharm. 2005;55(2):139-156.

21. Cappello B, De Rosa G, Giannini L, et al. Cyclodextrin-containing poly(ethyleneoxide) tablets for the delivery of poorly soluble drugs:Potential as buccal delivery system. Int J Pharm. 2006;319(2):63-70.

22. Yadav VR, Suresh S, Devi K, et al. Effect of Cyclodextrin Complexation of Curcumin on its Solubility and Antiangiogenic and Antiinflammatory Activity in Rat Colitis Model. AAPS PharmSciTech. 2009;10(3):752-762. 
23. Soliman AK, Ibrahim HK, Ghorab MM. Effect of different polymers on avanafil- $\beta$-cyclodextrin inclusion complex: in vitro and in vivo evaluation. Int J Pharm. 2016;512(1):168-177.

24. Weina Ma, Fugen Gu, Yi Wang, et al. Complexation of poorly aqueous soluble drug risperidone with hydroxypropyl- $\beta$-cyclodextrin enhances its dissolution. Journal of Chinese Pharmaceutical Sciences. 2015;24(1):47-53.

25. Mark David Bodley, Mino Rodolfo Caira, Lueta Ann Glintenkamp, et al. Inclusion complex of beta cyclodextrin and diclofenac, its preparation and use. USA; 1997.

26. Harry P Jones. Inclusion complex of $\beta$-cyclodextrin and digoxin. USA; 1985.

27. Mura P, Maestrelli F, Cirri M. Ternary systems of naproxen with hydroxypropyl- $\beta$-cyclodextrin and amino acids. Int $J$ Pharm. 2003;260(2):293-302.

28. Stella VJ, Rajewski RA. Cyclodextrins: Their future in drug formulation and delivery. Pharm Res. 1997;14(5):556-567.

29. Challa R, Ahuja A, Ali J, et al. Cyclodextrins in Drug Delivery: An Updated Review. AAPSPharmSciTech. 2005;6(2):329-357.

30. Giordano F, Novak C, Moyano JR. Thermal Analysis of Cyclodextrins and their inclusion Compounds. Thermochim Acta. 2001;380(2):123151.

31. Szejtli J. Introduction and general overview of cyclodextrin chemistry Chem Rev. 1998;98(5):1743-1753.

32. Warren Hirsch, Vojetch Fried, Lawrence Altman. Effect of cyclodextrins on sparingly soluble salts. J Pharm Sci. 1985;74(10):1123-1125.
33. Stella VJ, He Q. Cyclodextrins. Toxicol Path. 2008;36(1):30-42.

34. Nalluri BN, Chowdary KPR, Murthy KVR, et al. Inclusion Complexation and Dissolution Properties of Nimesulide and Meloxicam-hydroxypropyl- $\beta$-cyclodextrin Binary Systems. J Incl Phenom Macrocycl Chem. 2005;53(1):103-110.

35. Vyas A, Saraf S, Saraf S. Encapsulation of cyclodextrin complexed simvastatin in chitosan nanocarriers: A novel technique for oral delivery. $J$ Incl Phenom Macrocycl Chem. 2010;66(3):251-259.

36. Zheng Wang, Takashi Horikawa, Fumitoshi Hirayama, et al. Design and In-vitro Evaluation of a Modified-release Oral Dosage Form of Nifedipine by Hybridization of Hydroxypropyl-P-cyclodextrin and Hydroxypropylcellulose. J Pharm Pharmacol. 1993;45(11):942-946.

37. Hamza Yel S, Aburahma MH. Design and In vitro Evaluation of Novel Sustained-Release Double-Layer Tablets of Lornoxicam:Utility of Cyclodextrin and Xanthan Gum Combination. AAPS Pharm SciTech. 2009;10(4):1357-1367.

38. Gidwani B, Vyas A. Designing and evaluation of extended release matrix tablet containing altretamine-HP- $\beta-\mathrm{CD}$ inclusion complex. $J$ Incl Phenom Macrocycl Chem. 2015;83(3):401-409.

39. Miyake K, Arima H, Hirayama F, et al. (2000) Improvement of Solubility and Oral Bioavailability of Rutin by Complexation with 2-hydroxypropyl-3-cyclodextrin. Pharm Develop and Technol. 2000;5(3):399-407.

40. Choudhury S, Nelson KF. Improvement of oral bioavailability of carbamazepine by inclusion in 2 -hydroxypropyl- $\beta$-cyclodextrin. Int $J$ Pharm. 1992;85(1-3):175-180. 